\title{
Reporte de Caso Clínico y discusión de tema: Síndrome del opérculo Torácico
}

\author{
Report and Discussion of a Study Case: Thoracic \\ Outlet Syndrome
}

\section{Descrição do caso clínico e discussão do tema: síndrome do desfiladeiro torácico}

\author{
Celso Miguel Lemus Torres, MD., Esp * \\ Julián Enrique Cadena Ortiz, MD ** \\ Ángela Patricia Valle Lara, MD ** \\ Juan Camilo Mateus Jaime, MD *** \\ Jesús Eduardo Lemus Landínez ****
}

\begin{abstract}
Resumen
Introdución: El síndrome del Opérculo Torácico (SOT) es un grupo heterogéneo y potencialmente disfuncional de síndromes relacionados con compresión extrínseca de estructuras neurovasculares a su paso, desde el cuello hacia el miembro superior, por diferentes estructuras anatómicas congénitas o adquiridas, siendo más frecuente la compresión neurológica, ocasionando una variedad de sintomatología que varía según grado de compresión y estructura afectada, que en algunos casos hacen difícil su diagnóstico. Es una entidad mal diagnosticada teniendo en cuenta que no hay criterios diagnósticos establecidos; por eso, el examen físico es primordial para su sospecha clínica. El tratamiento inicial es conservador, siendo el tratamiento quirúrgico, una opción con múltiples procedimientos personalizados para cada tipo de paciente, según etiología. Objetivo: El propósito del artículo es realizar una discusión del caso clínico basada en la evidencia, para permitir un conocimiento general de esta patología, y comprender que hace parte de las causas de dolor en miembro superior, para así orientar al profesional de
\end{abstract}

la salud a realizar un diagnóstico oportuno y un tratamiento efectivo que disminuya el riesgo de complicaciones irreversibles. Resultados y conclusiones: Presentamos un caso clínico de una paciente Adulta Joven, con cuadro clínico caracterizado por síntomas neurovasculares, sincope, dolor torácico anterior izquierdo, y posterior dolor localizado en el miembro superior izquierdo, con sensación de masa en cuello, parestesias, disminución de la fuerza muscular, edema, palidez y frialdad del miembro. Se realizó diagnóstico radiológico de costilla cervical accesoria, confirmado con resonancia nuclear, se asoció a un síndrome de compresión del plexo braquial izquierdo, se realizó diagnóstico de síndrome de opérculo torácico izquierdo y fue llevada a cirugía ante la no mejoría con tratamiento conservador, se realizó un abordaje supraclavicular para resección de costilla cervical accesoria, escalenotomia, descompresión del plexo braquial y Arteria subclavia. Posterior, la paciente presentó mejoría notoria y remisión de los síntomas. [Lemus CM, Cadena JE, Valle AP, Mateus JC, Lemus JE. Reporte de Caso Clínico y discusión de tema: Síndrome del opérculo Torácico. MedUNAB 2015; 18 (2): 135-143]

* Ortopedia y Traumatología, Santa Casa de Bello Horizonte, Subespecialista en Hombro, codo y Mano, Santa Casa de Bello Horizonte, Bello Horizonte Brasil, Jefe Departamento Ortopedia y Traumatología Clínica La Riviera, Coordinador Médico Clínica La Riviera.

** Médico General, Universidad Autónoma de Bucaramanga, Médico General Clínica La Riviera.

*** Médico General, Universidad Pedagógica y Tecnológica de Colombia, Médico General Clínica La Riviera.

**** Estudiante de Medicina, Universidad Autónoma de Bucaramanga.

Correspondencia: Julián Enrique Cadena Ortiz. Calle 51 \#38-53 Clínica La Riviera, Bucaramanga, Santander. Correo electrónico: jcadenao@gmail.com. 
Palabras clave: Síndrome del Desfiladero torácico; Síndrome de la costilla cervical; Costilla cervical; Plexo braquial; Neuropatías del plexo braquial; Arteria subclavia; Vena subclavia.

\section{Abstract}

Introduction: Thoracic Outlet Syndrome - TOS - is a heterogeneous and potentially dysfunctional group of syndromes related to extrinsic compression of neurovascular structures from the neck to the upper limb for different congenital or acquired anatomical structures, being more frequent the neurological compression, causing a variety of symptoms that vary according to the degree of compression and affected structure that in some cases make it difficult to diagnose. Due to the lack of established diagnostic criteria, the physical exam is the most important element used to formulate a diagnosis. The initial treatment is conservative. The surgical treatment becomes an option with multiple personalized procedures for each type of patient according to the etiology. Objective: The purpose of the article is to discuss the clinical case based on the evidence allowing general knowledge of that pathology, and understanding the causes of pain in the upper limb, to therefore guide medical staff to perform a timely diagnosis and effective treatment that decreases the risk of irreversible complications. Results and conclusions: A study case of a young adult patient presenting neurovascular symptoms due to syncope, left anterior thoracic pain, localized pain in the upper limbs, sensation of lumps in the neck, paresthesia, decrease in muscular strength, edema, pallor and coldness of the limb was presented. A radiological diagnostic test in the accessory cervical rib was performed and later confirmed with a nuclear resonance. Thoracic Outlet Syndrome in the left side was diagnosed resulting in surgery. It was necessary to perform supraclavicular approach, scalenotomy, decompression of brachial plexus and subclavian artery. Later, the patient's health vastly improved. [Lemus CM, Cadena JE, Valle AP, Mateus JC, Lemus JE. Report and discussion of a study case: Thoracic Outlet Syndrome. MedUNAB 2015; 18 (2): 135-143]

Keywords: Thoracic outlet syndrome; Cervical Rib Syndrome; Cervical Rib; Brachial Plexus; Brachial Plexus neuropathies; Subclavian Artery; Subclavian Vein.

\section{Introducción}

El Síndrome de Opérculo torácico (SOT) es una entidad infrecuente, poco conocida, caracterizada por una compresión continua o intermitente de estructuras del paquete neurovascular - plexo braquial, arteria y vena subclavia - por anomalías anatómicas congénitas o adquiridas siendo más frecuente la compresión entre los músculos escalenos anterior y medio y la primera costilla (1-10). La sintomatología es variable y numerosa en estadios iniciales incluyendo síntomas neurológicos como cefalea, sincopes, parestesias, cervicalgia, dolor tipo anginoso o disconfort (1-10). Hay factores de riesgo asociados para el desarrollo del síndrome generados por

\section{Resumo}

Introdução: A Síndrome do desfiladeiro Toraxico (SOT) é um grupo heterogéneo e potencialmente disfuncional de síndromes relacionadas à compressão extrínseca de estruturas neurovasculares da passagem do pescoço para o membro superior por diferentes estruturas anatômicas congênitas ou adquiridas, sendo a compressão neurológica mais frequente, sua varieda sintomatología depende do grau de compressão e da estrutura afectada, o que em alguns casos podem dificultar o seu diagnóstico. É uma doença mal diagnosticada porque não há criterios estabelecidos para o diagnóstico; por esta razão o exame físico é fundamental na suspeita clínica. O tratamento inicial é conservador, sendo a opção cirúrgico uma intervenção com múltiplos procedimentos personalizados acordo com a etiologia do paciente. Objetivo: O objetivo deste artigo é realizar uma discussão do caso clínico com base em provas, que permita um conhecimento geral da doença, e entender que faz parte das causas de dor no membro superior permitindo orientar o profissional de saúde a fazer um diagnóstico oportuno e um tratamento eficaz a fim de reduzir o risco de complicações irreversíveis. Resultados e conclusões: O caso clínico estudado é de uma paciente jovem adulta com um quadro clínico caracterizado por sintomas neurovasculares por síncope, com dor toráxica anterior esquerdo e, em seguida, a dor localizada no membro superior esquerdo, com a sensação de massa no pescoço, parestesias, diminuição da força muscular, edema, palidez e frieza do membro. Foi realizado o diagnóstico radiológico costela cervical acessória, confirmado por ressonância nuclear associada à compressão do plexo braquial esquerda, o diagnóstico é da síndroma do desfiladeiro toxácica esquerdo. Como a paciente nao melhora com o tratamento recebido, é realizada a cirurgia supraclavicular para a realização de uma ressecção da costela cervical do acessória, escalenotomia, descompressão do plexo braquial e da artéria subclávia. Posteriormente, a paciente tem notável melhora e remissão dos síntomas. [Lemus CM, Cadena JE, Valle AP, Mateus JC, Lemus JE. Descrição do caso clínico e discussão do tema: síndrome do desfiladeiro torácico. MedUNAB 2015; 18 (2): 135-143]

Palavras-chave: Síndrome do Desfiladeiro Torácico; Síndrome da Costela Cervical; Costela Cervical; Plexo Braquial; Neuropatias do Plexo Braquial; Artéria Subclávia, Veia Subclávia.

predisposición congénita o adquirida, ya sea por traumas repetitivos (lesiones por hiperflexión o hipertensión de la columna cervical) o factores que produzcan espasmo crónico de la musculatura del cuello (1-10). Por la variedad de sintomatología su diagnóstico puede pasar desapercibido $\mathrm{o}$ asociarse con otro tipo de síndromes que generan dolor en miembro superior, tórax y cuello. El compromiso más frecuente es el nervioso; en los pacientes adolescentes el compromiso acompañado suele ser el vascular y el óseo, siendo necesario descartar malformaciones óseas vasculares, así como coagulopatías (9). El diagnóstico es principalmente clínico, siendo este un reto ya que no hay criterios diagnósticos específicos. Un buen examen físico y el conocimiento de los síntomas son necesarios para un diagnóstico oportuno, a través de la realización de las 
maniobras de provocación y reproducción de los síntomas $(3,8)$. Las ayudas diagnósticas como imágenes, estudios de neuroconducción, estudios vasculares son para confirmación del diagnóstico, evaluación de complicaciones o cuando hay diagnósticos diferenciales que requieran ser descartados (1,7-10). El tratamiento es debatido y variable según estructura afectada, etiología y progresión, siendo el manejo de primera línea conservador (1-10). El tratamiento quirúrgico es controversial. No hay criterios claros y específicos de inclusión, siendo el más frecuente la no resolución sintomática posterior al manejo conservador. Se incluyen otros criterios como anormalidad anatómica reconocida, compromiso vascular o nervioso asociado a atrofia muscular con mejoría hasta de un $90 \%$, siendo de mejor pronóstico y evolución los pacientes jóvenes y no fumadores (2-6). Las altas tasas de recaídas que pueden llegar hasta un $60 \%$ son secundarias a atrapamiento cicatricial, resección incompleta; sin embargo, es mucho menor la tasa de complicaciones asociadas hasta un $30 \%$, que van desde lesiones del plexo braquial y otras estructuras nerviosas así como complicaciones por lesiones pulmonares o vasculares, haciéndose necesario el conocimiento adecuado de la anatomía y sus variantes para evitar lesiones irreversibles $(3-5,10,11)$.

\section{Caso Clínico}

Paciente femenina de 22 años de edad, estudiante Universitaria, en seguimiento ambulatorio por cuadro clínico de aproximadamente 4 años de evolución caracterizado, inicialmente, por episodios sincopales con movimientos bruscos del cuello, posteriormente inició con sensación de masa y dolor en región supraclavicular izquierda con irradiación a tórax ipsilateral asociado a edema de la mano, palidez y frialdad intermitente; el dolor era continuo en intensidad de leve a moderada según escala subjetiva de dolor; manejado inicialmente con analgésico, antiinflamatorio oral y vitamina B. Con antecedentes de Acné, Rinitis Alérgica con hipertrofia de cornetes, y antecedente familiar de primer grado Cáncer de Colon, sin antecedentes quirúrgicos, o alérgicos asociados. En la revisión por sistemas se encontró asociación con sintomatología ansiosa y magnificación de la sintomatología, por lo que se solicitó valoración por psicología.

Por persistencia de la sintomatología se toma ecografía de tejidos blandos de cuello y tomografía axial computarizada simple y contrastada, ambos exámenes dentro de límites normales. La paciente continuó con dolor moderado en la escala subjetiva de dolor por al menos un año más, por lo cual consultó nuevamente y se tomó una nueva ecografía de tejidos blandos cervicales donde se reportó una masa de consistencia dura en región supraclavicular izquierda con imagen ecogénica, con sombra acústica lineal y alargada de etiología a establecer, se tomó una radiografía de columna cervical que reportó hallazgo de costilla cervical accesoria izquierda (Figura 1).

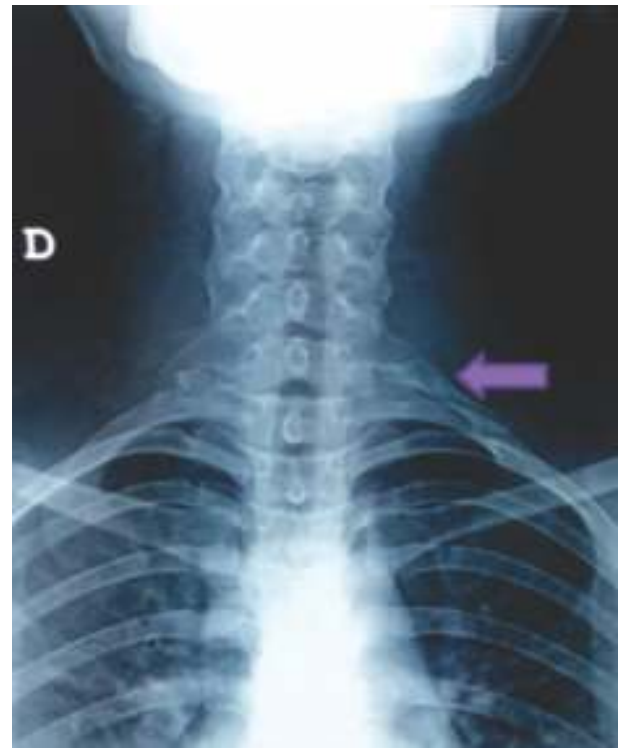

Figura 1. Radiografía de columna cervical, la flecha en color indica la costilla cervical accesoria izquierda, sin evidencia contralateral - Tomado de historia clínica con autorización de paciente.

Al examen físico pruebas de desencadenamiento, Adson, costoclavicular y prueba de hiperabducción positivas, triada de Selmonosky positiva asociada a disminución de fuerza muscular del miembro superior izquierdo, posterior se solicitó una resonancia nuclear magnética la cual confirmó la presencia de costilla cervical accesoria con compresión de la porción distal de troncos primarios del plexo braquial; posteriormente se toma electromiografía y neuroconducción que reporta dentro de límites normales. Por diagnóstico de Síndrome de opérculo torácico secundario a costilla cervical accesoria sin mejoría sintomática es valorada y programada en conjunto por cirugía del tórax y ortopedia de miembro superior para resección quirúrgica.

Se realiza el procedimiento quirúrgico con abordaje supraclavicular izquierdo (Figura 2), hallazgos intraoperatorios

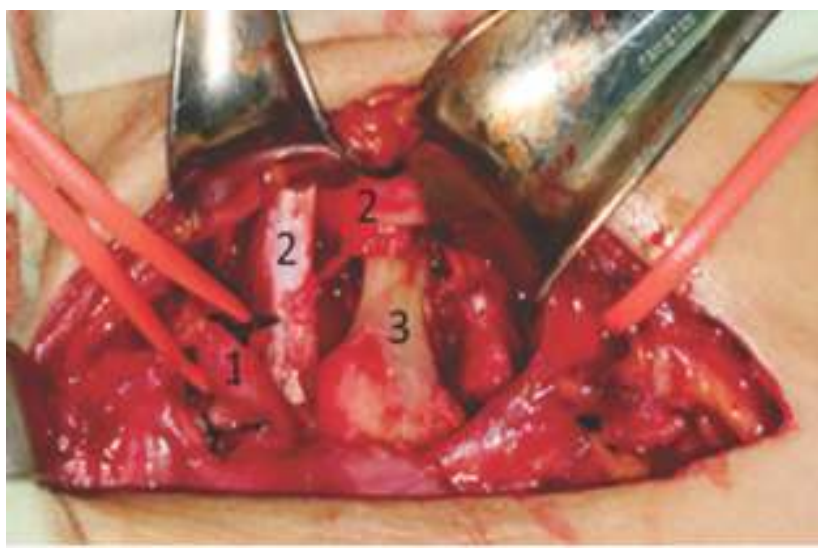

Figura 2. Imagen intraoperatoria: 1. Arteria Subclavia 2. Plexo Braquial 3. Costilla Cervical accesoria. Tomada con consentimiento y autorización previa de la paciente. 


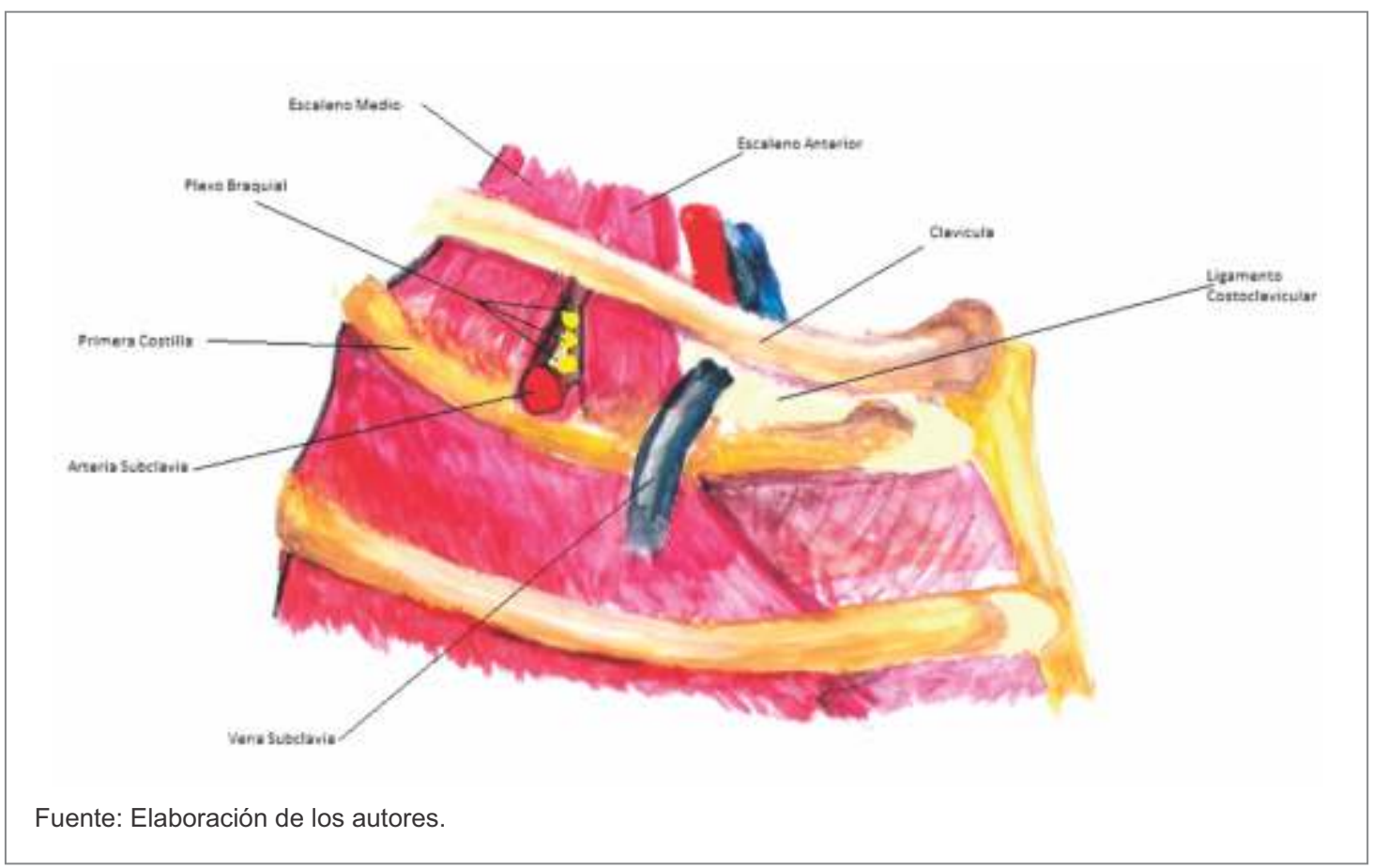

Figura 3. Esquema de la anatomía del opérculo torácico.

de costilla cervical accesoria completa con exostosis de la misma articulándose con la apófisis transversa de $\mathrm{C} 7$ y primer arco costal, compresión de la arteria subclavia y del plexo braquial en los troncos medio y superior, con fascias anómalas del escaleno anterior y medio. Se realizó neurolisis y descompresión del plexo braquial con posterior división de la fascia del escaleno anterior y medio (escalenotomia), resección de la costilla cervical y descompresión de la arteria subclavia. La paciente fue citada a control postquirúrgico a los 8 días con evolución satisfactoria con mejoría sintomática importante. Posterior a nuevo control tres meses después, la paciente comentó resolución total de la sintomatología considerándose evolución clínica satisfactoria.

La paciente vuelve a control 10 meses después de la cirugía, refiriendo una mejoría absoluta de la sintomatología neurológica y vascular. Hasta el momento la paciente no ha presentado complicaciones médico quirúrgicas, y su sintomatología no ha recurrido en más de 1 año posterior al procedimiento quirúrgico. Se da de alta por cirugía de mano, y se explica que en caso de recurrencia de la sintomatología solicite nuevo control.

\section{Discusión}

El síndrome del opérculo torácico (SOT), es un síndrome mal diagnosticado y raro, que consiste en la compresión o irritación extrínseca, posicional, continúa o intermitente, de las estructuras neurovasculares (plexo braquial, arteria subclavia y vena subclavia) del cuello mientras pasan por el opérculo torácico hacia la axila y el brazo. Puede comprometer la extremidad superior, el cuello, el tórax, los hombros y la cabeza (1-23).

Epidemiologia: El SOT es responsable del 5 al 10\% de los síntomas dolorosos de la extremidad superior (6). Afecta al $8 \%$ de la población general, con una frecuencia de 3-80 casos por 1.000 individuos y una incidencia desconocida $(5$, 10, 19). Sin embargo, esta ha sido estimada en 5:100.000 casos por año en el Reino Unido (3). Es más común en las mujeres que en los hombres con una relación 3-4:1 (4-6, 10). Los casos más frecuentes se dan en los jóvenes y adultos entre los 25 a los 40 años (4-7). Los síntomas neurogénicos son los más frecuentes, siendo alrededor de 90-95\% de todos los casos, seguido de la sintomatología venosa en un $5-10 \%$ y arterial en $<1 \%$ aproximadamente ( 1 $3,8,10,16,18-20)$. La costilla cervical es la causa más común, tiene una incidencia del 0,2 al 1\% de la población, 50 al $80 \%$ son bilateral y solo el 10 al $20 \%$ producen síntomas, tiene una relación de 2:1 en las mujeres versus los hombres $(8,10,12,20)$. En Colombia no hay estudios clínicos epidemiológicos donde se pueda verificar incidencia, prevalencias o factores epidemiológicos asociados.

Anatomía: El opérculo torácico está delimitado en la parte superior por el músculo subclavio y la clavícula, anteriormente por el músculo escaleno anterior, y posterior por el músculo escaleno medio $(2,4,10,16,20)$. Está compuesto por 3 espacios potenciales (Figura 3 y 4 ): 


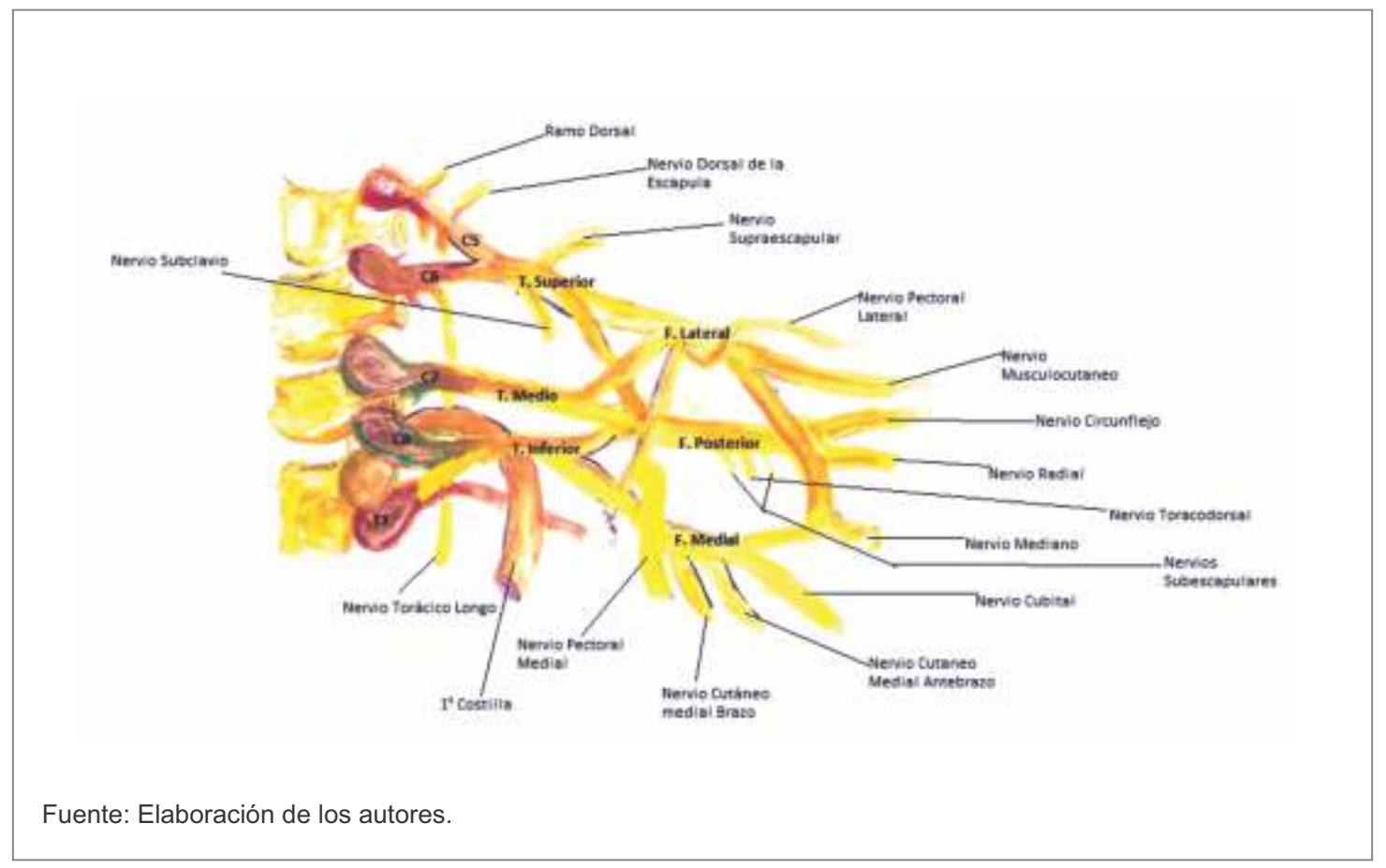

Figura 4. Esquema Plexo Braquial y Nervios del Miembro Superior.

Triángulo interescalénico, lo comprende la unión del músculo escaleno anterior, el músculo escaleno medio y la clavícula. Es atravesado por la arteria subclavia y los troncos primarios del plexo braquial. Queda por fuera la vena subclavia. Espacio costoclavicular, delimitado anteriormente por el músculo subclavio, superiormente por el tercio medio de la clavícula, posteromedial por la primera costilla y posterolateral por el borde superior de la escápula; contiene los vasos subclavios y los troncos secundarios del plexo braquial. El Espacio retropectoral menor o subcoracoideo está limitado anteriormente por el pectoral menor, superior por la apófisis coracoides y posterior por la pared torácica y la escápula, es atravesado por los nervios del plexo braquial y por la porción distal de la arteria subclavia y de la vena subclavia $(2,4,6,7,10,16$, 19-21).

Etiología: El SOT tiene amplio espectro de etiologías; sin embargo, la literatura las ha encerrado en 3 grandes grupos; las anormalidades óseas, las anormalidades de tejidos blandos y las anormalidades posturales. Dentro de las anormalidades óseas se encuentran las costillas cervicales, mega apófisis transversa de $\mathrm{C} 7$, anormalidades de la primera costilla, no uniones o pseudoartrosis de la clavícula, callos hipertróficos de la clavícula o tumores. Por otro lado, están las anormalidades de tejidos blandos, donde se encuentran la hipertrofia muscular cervical (músculos escaleno anterior, escaleno medio, pectoral menor y subclavio) o las bandas fibrosas (ligamentos costocoracoideo, membrana costoclavicular) o anomalías musculares congénitas $(1-4,6,7,9,11,12,16,18,19,21)$.
Manifestaciones Clínicas: el SOT está compuesto por tres formas usuales. La primera, es el SOT neurogénico (SOTN), que comprende la sintomatología neurológica del síndrome; la segunda, es el SOT venoso (SOTV), que produce síntomas venosos puros, como trombosis de la vena subclavia, y por último, está el SOT arterial (SOTA), el cual es extremadamente raro, y se manifiesta con sintomatología arterial pura secundaria a trombosis arterial o aneurismas arteriales de la arteria subclavia. Sin embargo, el SOT puede manifestarse con uno solo de los síntomas, o se pueden combinar (Tabla 1) $(1,3,5-10,15$, $16,18-20)$.

Examen Semiológico: en cuanto al examen físico, se debe enfatizar que el SOT es un diagnóstico clínico, basado en una correcta recolección de datos clínicos. Por lo cual, un buen examen físico debe iniciarse por una inspección del cuello, hombros, y extremidades superiores. Se debe examinar el color, trofismo muscular, edema, temperatura y las uñas. También, debe realizarse un examen neurológico meticuloso, inspeccionando sensibilidad, fuerza muscular, reflejos y otros signos neurológicos (signos de tinnel o fahlen) $(1,4,8,9,18,19)$.

Para realizar un diagnóstico clínico correcto, se debe realizar una serie de pruebas o test que durante años se han descrito y aprobado para identificar el diagnóstico de SOT, los cuales se describirán a continuación $(1-9,18,19)$.

1. Test de Adson: consiste en la toma del pulso radial en el brazo a evaluar, con una hiperextensión cervical y 
Tabla 1. Sintomatología del Síndrome de Opérculo Torácico, SOT.

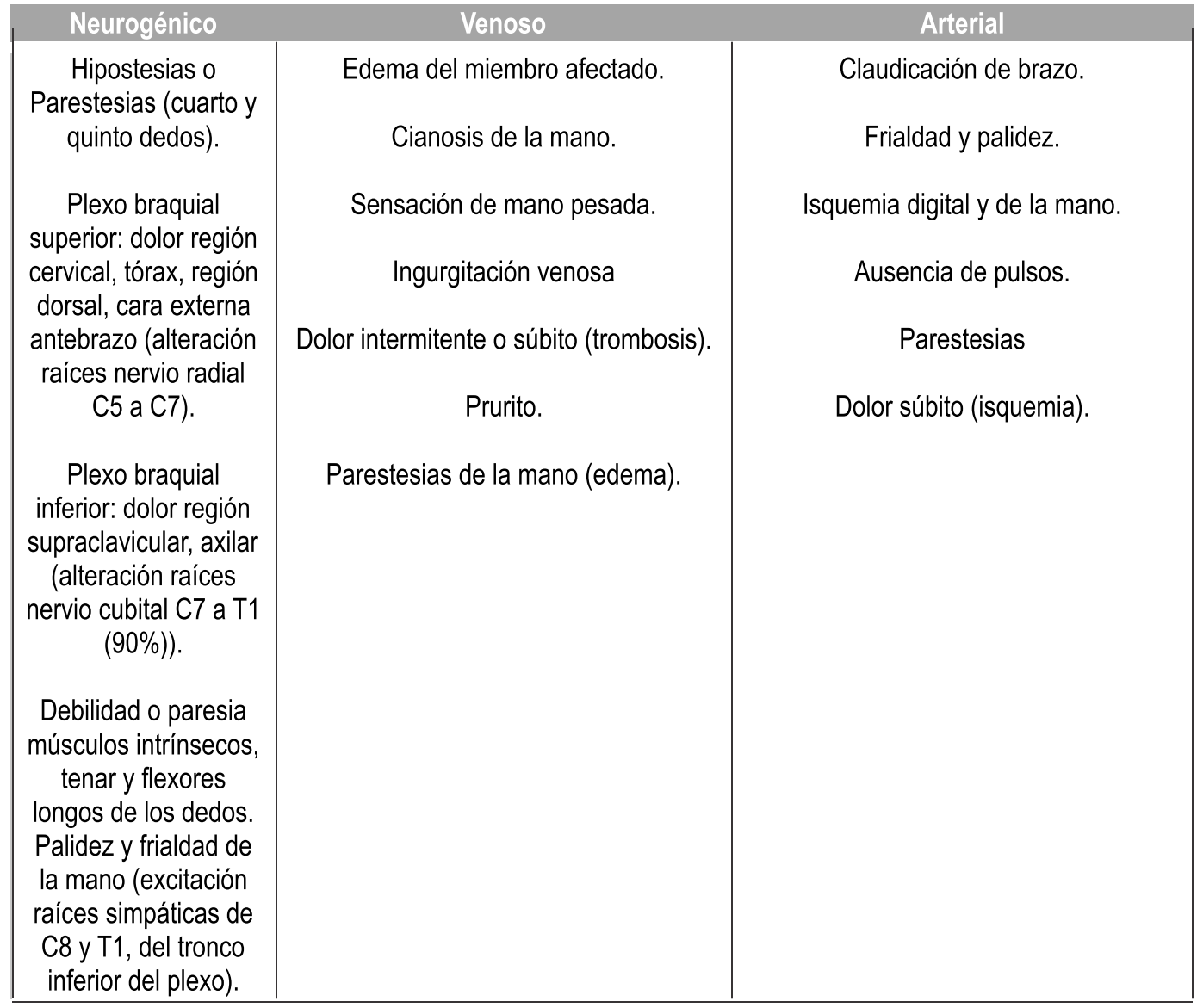

Fuente: Elaboración de los Autores.

llevando en rotación y latero flexión hacia el lado afectado. La prueba es positiva si hay ausencia o disminución del pulso. (triángulo interescalénico) $(1-8$, $16,18,9)$.

2. Test de hiperabducción de wrigth: se localiza el pulso radial, se lleva el brazo a evaluar en hiperabducción y en rotación externa del hombro con el codo en extensión. El test es positivo, si el pulso radial disminuye o está ausente. (Espacio retropectoral menor o subcoracoideo) $(1-8,18,19)$.

3. Test de Edén: paciente en sedestación, se localiza el pulso radial, se lleva el brazo a evaluar en rotación externa, se realiza presión sobre la clavícula para hacerla descender. Si hay disminución del pulso el test será positivo. (espacio costoclavicular) (1-8).

4. Triada de Selmonosky: es un test simple que detecta, las más pequeñas compresiones neurovasculares presentes y consiste en: Evaluar la debilidad en la abducción, aducción, fatiga u oposición del quinto dedo. Evaluar la presencia de parestesias, fatiga, cansancio asociado o no, con palidez de la mano al realizar la elevación de la extremidad superior afectada. Evaluar la sensibilidad de ambas áreas supraclaviculares al realizar su dígitocompresión con el pulgar, la cual debe ser dolorosa del lado comprometido (9).
5. Test de Roos: paciente coloca los 2 brazos con abducción de $90^{\circ}$, con los codos flexionados a $90^{\circ}$. Posteriormente, las manos se abren y se cierran repetidamente por un periodo de aproximadamente 3 minutos. Es Positivo cuando ocasiona disconfor o fatiga muscular, lo que ocasiona que el paciente no sea capaz de continuar con la maniobra en el lado afectado $(18,19)$

Ayudas Diagnósticas: Radiografia de tórax y cervical, para descartar o confirmar anormalidades óseas $(2-10,16$, 18, 19). Por otro lado, está la Tomografía Axial computarizada (TAC), ofrece una mejor información anatómica, en especial cuando se sospecha de una etiología de origen óseo $(5,6,9,10,18-20)$. A su vez, la Resonancia Nuclear Magnética (RNM) permite una adecuada valoración de los tejidos blandos, especialmente del plexo braquial $(3,5,6,9,10,16,18-20)$. La Electromiografía $(E M G$,) en estudios fue negativa en pacientes con SOT. Sin embargo, se considera útil para descartar otras anormalidades neurológicas $(3,7-10,16,18,20)$. La Arteriografia, su utilidad es muy limitada, y solo debe ser utilizada cuando un paciente presenta síntomas y signos de insuficiencia e isquemia arterial $(2,6-10,16)$.Existen otros métodos diagnósticos, como el Bloqueo interescalénico 
Tabla 2. Diagnóstico Diferencial del Síndrome de Opérculo Torácico.

\begin{tabular}{l}
\hline Diagnóstico Diferencial Síndrome de Opérculo Torácico \\
\hline Discopatía Cervical \\
\hline Síndrome de Túnel Carpiano y Cubital \\
\hline Bursitis y Tendinitis del Hombro \\
\hline Espondilitis Cervical \\
\hline Esclerosis Múltiple \\
\hline Tumor Medular \\
\hline Angina Pectoris \\
\hline Síndrome Pectoralis Minor \\
\hline Vasculitis miembros superiores \\
\hline Osteoartritis glenohumeral o acromioclavicular \\
\hline
\end{tabular}

Fuente: Elaboración de los Autores.

diagnóstico, el cual consiste en el bloqueo anestésico temporal que paraliza el músculo escaleno anterior y permite el descenso de la primera costilla, lo que admite descomprimir el SOT. Es uno de los métodos diagnósticos más efectivos para diagnóstico y confirmación de SOT secundario a hipertrofia o espasmos del músculo escaleno anterior $(5,10,16,18)$.

Diagnóstico Diferencial: es importante mencionar que el SOT puede ser mimetizado por múltiples patologías y síndromes, por lo cual la clínica, y las ayudas diagnósticas son importantes para realizar un diagnóstico diferencial (Tabla 2) $(16,19)$.

Tratamiento: Se debe realizar un manejo multidisciplinario, enfocado en tres objetivos principales:

1. Disminución en la compresión neurovascular.

2. Manejo del dolor y control sintomático.

3. Mejorar calidad de vida.

Las opciones de tratamiento son de gran variedad, desde procedimientos conservadores, hasta mínimamente invasivos o procedimientos quirúrgicos complejos que deben ser personalizados para cada paciente $(2-7,10)$. Si no hay mejoría clínica, el tratamiento quirúrgico está indicado cuando hay falla en manejo conservador $(2-7,10,19,20)$.

Tratamiento conservador: el manejo de primera línea cuando no hay compromiso neurovascular involucra además de medicación, terapia física y ocupacional, educación para modificación de estilos de vida hasta procedimientos mínimamente invasivos dirigidos al control del dolor (2-7). Se espera una respuesta a estos entre los 3 y 6 meses de tratamiento. Hay que tener en cuenta que no siempre el manejo conservador es curativo, sin embargo, las tasas de éxito y mejoría del dolor llegan alrededor del $60 \%$ hacia el segundo y tercer mes de tratamiento $(2-7,10,16,18-23)$.

Educación al paciente: modificaciones en factores ambientales y en el estilo de vida, como posturas que disminuyan el dolor, siendo principal la modificación de la postura al caminar, sentarse o dormir que disminuyen considerablemente los síntomas, se incluye además la pérdida de peso, disminución del peso de las mamas con cambio de sostén y realización de ejercicios para fortalecimiento de musculatura cervical $(5,18,22)$.

Medicación: la terapia va dirigida al control del dolor con medicación analgésica tipo opioide, antiinflamatorios, relajantes musculares, estabilizadores de membrana celular, hasta antidepresivos tricíclicos, inhibidores de la recaptación de serotonina y anticoagulación en pacientes con compromiso vascular arterial o venoso con presencia de trombosis o isquemia crítica $(5,10,18,22)$.

Terapia física y ocupacional: se incluyen masajes relajantes para disminuir espasmo, los ejercicios de Peet que mejoran la postura e imbalance cervicoescapular y terapia ocupacional con minimización del trabajo y técnicas de protección. Se espera que con 10 a 20 sesiones haya mejoría sintomática y del dolor $(2-7,22)$.

Procedimientos mínimamente invasivos: se realiza aplicación de medicamentos en el espacio interescalénico y en la musculatura cervicotorácica para manejo del dolor, incluyendo medicamentos como anestésicos locales, corticoides y toxina botulínica, a través de marcación anatómica o guiada por TAC, ultrasonido, fluoroscopio o por electromiografía cuando se desea realizar bloqueos en nervios específicos $(5,10)$. La toxina botulínica recién descubierta ha sido utilizada en múltiples patologías que requieran específicamente de disminución de la actividad 
muscular a través de la inhibición de los canales de calcio dependientes de acetil colina de forma presináptica en la placa neuromuscular. El espasmo crónico produce cambio en la histología produciendo fibrosis muscular. La toxina botulínica al reducir la actividad muscular disminuye la injuria, reduciendo el dolor y la inflamación, con actividad neurolisis química, reportándose disminución de hasta 29\% a los tres meses de aplicación $(5,10,23)$. Su duración aproximada es de 3 a 6 meses, tiempo que dura el efecto de la toxina $(5,10)$. Su uso repetitivo está limitado ya que genera anticuerpos que disminuyen su efecto terapéutico y duración de la acción con dosis siguientes $(5,10)$. Se ha visto una mayor reducción sintomática con el uso de toxina botúlica en combinación con infiltraciones con anestésicos y corticoides, siendo el $64 \%$ comparado con $18 \%$ la tasa de mejoría del dolor respectivamente $(5,10,23)$.

Otros procedimientos: se incluye la Estimulación Nerviosa Transcutánea (por sus siglas en inglés, TENS) a través de ondas de choque para el manejo del dolor $(5,9$, $10)$.

Tratamiento quirúrgico: va dirigido según etiología y compromiso, controversial ya que no hay indicaciones ni criterios unificados ni una técnica quirúrgica específica; sin embargo, se tienen en cuenta las siguientes condiciones: 1 . No respuesta al manejo conservador al menos durante 3 meses; 2. Compromiso motor / sensitivo; 3. Claudicación vascular; 4. Anomalía anatómica reconocida 5. Disminución en las actividades de la vida diaria $(4,17,19)$. También hay que considerar factores predictores de éxito o fracaso del procedimiento: 1. Edad avanzada mayor a 40 años; 2. Fumador activo; 3. Larga duración de sintomatología con necesidad de uso de opioides; 4. Comorbilidades con síndromes dolorosos del hombro cuello (5). Existen muchas modalidades quirúrgicas dirigidas ya sea por preferencia quirúrgica o por anatomía comprometida con intervención de múltiples especialidades, siendo los principales procedimientos dirigidos a descompresión de estructuras afectadas: resección primera costilla, Resección costilla cervical, resección bandas fibróticas, escalenectomia anterior y media, neurolisis del plexo braquial hasta tenotomía del pectoral menor $(2-6,11$, $12,14,17)$. Cuando hay compromiso vascular ya sea por aneurismas, trombosis o isquemia crítica son aplicables procedimientos por vía endovascular o abierta según preferencia o compromiso mayor de otras estructuras que requiera de reparación abierta como son trombectomias, trombolisis mecánica, angioplastia para manejo de estenosis o aneurismas arteriales o venosos generalmente de la subclavia $(4-6,9,12)$. Son conocidas cuatro vías de ingreso: transaxilar, supraclavicular, subclavicular y posterior.

1. Transaxilar: Considerada por muchos la mejor opción quirúrgica descrita por Ross y Owens (2, 4, 18, 21, 22), pues encamina a resecar la estructura ósea que constituye la raíz del problema (4), la primera costilla por lo cual tiene mayor tasa de éxito (92\%), además de tener un buen resultado estético. Sin embargo, es la que mayor tasa de complicaciones reporta $(3,4,17)$. Además de ser una vía de difícil acceso para reparos vasculares, requiere buena experiencia para evitar lesiones de estructuras vasculares y nerviosas vecinas $(3,5,22)$. Tiene una tasa de recurrencia de aproximadamente el $20 \%$ de los casos (17).

2. Supraclavicular: Vía de preferencia por muchos ya que permite realizar resección de la primera costilla, además de tener un buen acceso para los reparos vasculares $(3,12,18,21,22)$. Es la vía indicada cuando hay compromiso de plexo braquial $(3,4,18,21,22)$. Tiene la menor tasa de complicaciones y un menor tiempo quirúrgico $(3,11)$. Hasta un $96 \%$ de los pacientes tienen éxito $(3,10-12,23)$. Hay mayor riesgo de lesión de plexo braquial y de estructuras vasculares, así como resultado estético con cicatriz visible $(3,4$, 10-12).

3. Subclavicular: Tiene una indicación específica en casos de opérculo torácico bilateral para exposición combinada $(4,5)$.

4. Posterior: Brinda exposición limitada e implica resección muscular amplia. Se utiliza solamente en reintervenciones $(4,5,18)$.

Se habla en general de que no hay diferencias significativas ni en la morbimortalidad ni en la tasa de éxito entre las vías transaxilar y supraclavicular, esta última cercana al $90 \%$ en el post-operatorio y hasta $70 \%$ a los 10 años de seguimiento ( $3,10-12)$.Se conoce además que los pacientes adolescentes tienen una respuesta más favorable y una menor tasa de complicaciones comparados con los adultos $(13,14)$.

Complicaciones: Además de las propias de cualquier procedimiento quirúrgico y las asociadas a la anestesia, se presentan en general lesiones a estructuras vecinas ya sean nerviosas, vasculares, y en región torácica.

1. Lesión nerviosa: Puede presentarse por lesión directa o por tracción secundaria a la manipulación o movilización excesiva del cuello y miembro superior con tasas reportadas del 1\% del plexo braquial, y lesiones del nervio frénico siendo del 6 al 12\% lesiones transitorias $(10-12,18)$. La lesión se ve más frecuente en la vía supraclavicular $(3,4,10,16)$. La lesión del nervio torácico largo se reporta en 1\% producida durante la escalenectomia (4, 10). También puede lesionarse el nervio frénico. Estas lesiones tiende a ser transitorias $(9,16)$.

2. Lesión Vascular: La lesión de vena y arteria subclavia son más frecuentemente reportadas, hasta en un 12 a $15 \%$ con necesidad posterior de reparo vascular $(9,18)$.

3. Lesiones torácicas: Se reportan tasas de 2 a $15 \%$ en neumotórax $(3,10)$, lesión pulmonar y mala mecánica ventilatoria posterior a procedimiento con necesidad de inicio de terapia respiratoria con incentivo temprano $(4,9,18)$ 
4. Recurrencia: Se ha descrito una tasa de recurrencia de aproximadamente $20-63 \%$ en los casos de SOT (17, 18, 23). Sin embargo, hacen falta estudios longitudinales, y epidemiológicos que soporten esta evidencia, ya que la mayoría de referencias es de series de casos.

\section{Conclusiones}

Como se expuso en el caso clínico, el síndrome de opérculo torácico es una patología poco frecuente, prevalente en mujeres jóvenes, con una amplia variedad de síntomas y signos, que hacen de este, un síndrome complejo y de difícil diagnóstico que amerita un conocimiento previo para su oportuno diagnóstico y un tratamiento efectivo que disminuya el riesgo de complicaciones irreversibles. Se ha de tener en cuenta que el objetivo del tratamiento inicial debe ser conservador para reducir los síntomas, y posteriormente si no hay presencia de mejoría, debe brindarse la alternativa quirúrgica, realizando descompresión quirúrgica, en este caso por vía supraclavicular o transaxilar, los cuales son los más aceptados por sus buenos resultados a largo plazo y menor tasa de complicaciones. Por tanto, se hace un llamado a los profesionales de salud, primordialmente a los médicos en formación, médicos generales y especialistas, para que tengamos en cuenta el síndrome de opérculo torácico, que aunque si bien es una patología rara y desconocida, su sintomatología puede desencadenar alteraciones funcionales importantes, que pueden llegar incluso a ser definitivas.

\section{Referencias}

1. Thompson R. Challenges in the treatment of thoracic outlet syndrome. Tex Heart Inst J. 2012; 39 (6): 842-843.

2. Lindgren K. Thoracic outlet syndrome. Int Musculoskelet Med. 2010; 32 (1): 17-24.

3. Campi P, Scotti Celeste, Gerevini S. Surgical treatment of thoracic syndrome in Young adults: single centre experience with minimun three-year follow up. Int Ortho. 2011; 35: 1179-1186.

4. Alvarez D, Guzman F, Vargas F. Síndrome del opérculo torácico. Rev Colomb Anestesiol. 1992; 20 (1): 55-62.

5. Pritzlaff S, Carinci A. Neurogenic thoracic outlet syndrome: An often overlocked but treatable condition. J Fam Pract. 2013; 32 (9): 16-21.

6. Márquez JC, Acosta M, Uribe J. Síndrome del opérculo torácico: Reporte de caso. Rev Colomb de Radiol. 2009; 20(4): 2803-2805.
7. Ashfag H, Sajad H, Showkat J, Zahida R, Shahnawaz H, Irfan J. Thoracic outlet syndrome, anatomical and surgical perspective. JK Science. 2011; 13 (2): 57-60.

8. Sanders R, Hammond S, Rao N. Diagnosis of thoracic outlet syndrome. J Vasc Surg. 2007; 46 (3): 601-604.

9. Selmonosky C, Poblete R. El diagnóstico del síndrome del opérculo torácico. Mitos y realidades. Rev Chil Cir. 2008; 60 (3): 255-261.

10. Boezaart A, Haller A, Laduzenski S, koyyalamudi V, Ihnatsenka B, Wrigth T. Neurogenic thoracic outlet syndrome: A case report and review of the literature. Int J Shoulder Surg. 2010; 4 (2): 27-35.

11. Yaseen Z, Baram A. Neurogenic thoracic outlet syndrome treatment by the supraclavicular approach. Assian Cardiovasc ThoracAnn. 2014; 22 (2): 193-196.

12. Chang K, Likes K, Davis K, Demos J, Freischlag J. The significance of the cervical ribs in thoracic outlet syndrome. J of Vasc Surg. 2013; 57 (3): 771-775.

13. Chang K, Graf E, Davis K, Demos J, Roethle T, et al. Spectrum of thoracic outlet syndrome presentation in adolescent. Arch Surg. 2011; 146 (12): 1383-1387.

14. Caputo F, Wittenberg A, Veuri C, Driskill M, Earley J, Rastogi R, Emery V, Thompson R. Supraclavicular decompression for neurogenic thoracic outlet syndrome in adolescent and adult populations. J Vasc Surg. 2013; 57 (1): 149-157.

15. Povlsen B, Hansson T, Povlsen SD. Treatment for thoracic outlet syndrome. Cochrane Database Syst Rev. 2014; 11: DOI: 10.1002/14651858.CD007218.pub3.

16. Sanders R, Hammond S, Rao N. Thoracic Outlet Syndrome ARiview. Neurologist. 2008; 14 (6): 365-373.

17. Dalbayrak S. et al. Supraclavicular Surgical Approach for Thoracic Outlet Syndrome. Turk Neurosurg. 2014; 24 (6): 867-872.

18. Kuhn J, Lebus G, Bible J. Thoracic Outlet Syndrome. J Am Acad Orthop Surg. 2015; 23: 222-232.

19. Stewman C, Vitanzo P, Hardwood M. Neurologic Thoracic Outlet Syndrome: Summarizing a Complex History and Evolution. Curr Sports Med Rep. 2014; 13 (2): 100-106.

20. Singh VK, Jeyaseelan L, Kyriacou S, et al. Diagnostic value of magnetic resonance imaging in thoracic outlet síndrome. Journal of Orthopaedic Surgery. 2014; 22 (2): 228-231.

21. Nichols A. Diagnosis and Management of Thoracic Outlet Syndrome. Curr Sports Med Rep. 2009; 8 (5): 240-249.

22. Brooke B, Freischlag J. Contemporary management of thoracic outlet syndrome. Curr Opin Cardiol. 2010; 25: 535-540.

23. Murovic J, Kim D, Kim S, Kline D. Thoracic Outlet Syndrome: Part I A Riview of the Recent Literature. Neurosurg Q. 2007; 17 (1): 1-12. 